This item was submitted to Loughborough's Research Repository by the author.

Items in Figshare are protected by copyright, with all rights reserved, unless otherwise indicated.

\title{
Symbolic arithmetic knowledge without instruction
}

PLEASE CITE THE PUBLISHED VERSION

http://www.nature.com/nature/journal/v447/n7144/full/nature05850.html

PUBLISHER

(C) Nature Publishing Group

VERSION

AM (Accepted Manuscript)

LICENCE

CC BY-NC-ND 4.0

REPOSITORY RECORD

Gilmore, Camilla K., Shannon E. McCarthy, and Elizabeth S. Spelke. 2019. "Symbolic Arithmetic Knowledge Without Instruction". figshare. https://hdl.handle.net/2134/8760. 
This item was submitted to Loughborough's Institutional Repository (https://dspace.lboro.ac.uk/) by the author and is made available under the following Creative Commons Licence conditions.

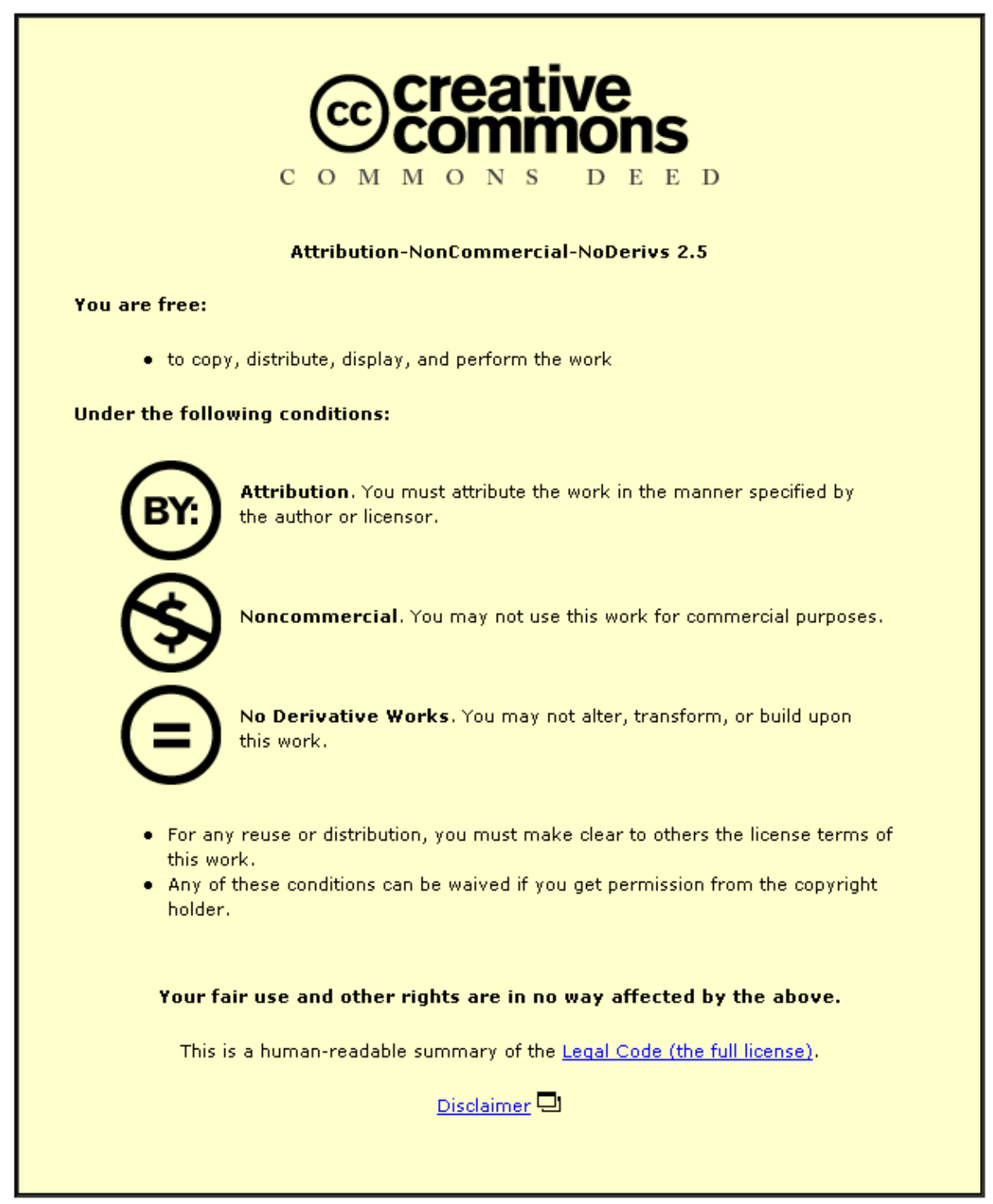

For the full text of this licence, please go to: http://creativecommons.org/licenses/by-nc-nd/2.5/ 
Symbolic Arithmetic Knowledge Without Instruction

Camilla K. Gilmore, Shannon E. McCarthy \& Elizabeth S. Spelke

This paper was published in Nature (2007), 447, 589-591

DOI:10.1038/nature05850 
Symbolic Arithmetic Knowledge Without Instruction

Camilla K. Gilmore ${ }^{1}$, Shannon E. McCarthy ${ }^{2}$ \& Elizabeth S. Spelke ${ }^{2}$

${ }^{1}$ Learning Sciences Research Institute, University of Nottingham, Wollaton Road, Nottingham NG8 1BB, UK; ${ }^{2}$ Department of Psychology, Harvard University, 33 Kirkland Street, Cambridge MA 02138, USA.

Symbolic arithmetic is fundamental to science, technology, and economics, but its acquisition by children typically requires years of effort, instruction, and drill ${ }^{\mathbf{2}}$. When adults perform mental arithmetic, they activate nonsymbolic, approximate number representations, ${ }^{34}$ and their performance suffers if this nonsymbolic system is impaired ${ }^{5}$. Nonsymbolic number representations also allow adults, children, and even infants to add or subtract pairs of dot arrays and to compare the resulting sum or difference to a third array, provided that only approximate accuracy is required ${ }^{6-10}$. Here we report that young children, who have mastered verbal counting and are on the threshold of arithmetic instruction, can build on their nonsymbolic number system to perform symbolic addition and subtraction ${ }^{11}$ 15. Children across a broad socio-economic spectrum solved symbolic problems involving approximate addition or subtraction of large numbers, both in a laboratory test and in a school setting. Aspects of symbolic arithmetic therefore lie within the reach of children who have learned no algorithms for manipulating numerical symbols. Our findings help to delimit the sources of children's difficulties learning symbolic arithmetic, and they suggest ways to enhance children's engagement with formal mathematics.

We presented children with approximate symbolic arithmetic problems in a format that parallels previous tests of non-symbolic arithmetic in preschool children ${ }^{89}$. In the first experiment, five- to six-year-old children were given problems such as "If you had twenty-four stickers and I gave you twenty-seven more, would you have more 
or less than thirty-five stickers?'. Children performed well above chance $\left(65.0 \%, t_{19}=\right.$ $2.77, P=.012)$ without resorting to guessing or comparison strategies that could serve as alternatives to arithmetic. Children who have been taught no symbolic arithmetic therefore have some ability to perform symbolic addition problems.

Children's performance nevertheless fell short of performance on non-symbolic arithmetic tasks using equivalent addition problems with numbers presented as arrays of dots and with the addition operation conveyed by successive motions of the dots into a box $\left(71.3 \%\right.$ correct, $\left.F_{1,34}=4.26, P=.047\right){ }^{8}$ Some children appeared to be confused by the wording of the problems; earlier experiments using even more complex wording had yielded chance performance in children of this age ${ }^{8}$. Accordingly, a second experiment tested children's understanding of symbolic addition with simpler questions accompanied by visual displays (Figure 1a). Children answered questions of the form "Sarah has fifteen candies and she gets nineteen more, John has fifty-one candies. Who has more?" Children's performance was as accurate $\left(73.3 \%, t_{19}=6.40, P=4 \times 10^{-6}\right)$ as the performance of children in past research given such problems in nonsymbolic form ${ }^{8}$ (Figure 2a), and again did not depend on guessing strategies involving comparisons between pairs of arrays.

The above experiments were conducted in a quiet laboratory setting and focused on children living in a relatively wealthy and highly educated community. To investigate their generality, we used the method of the preceding experiment to assess the symbolic arithmetic performance of children from a low- to middle-socioeconomic community, tested in their kindergarten classrooms. These 5- to 6-year-old children also performed well above chance $\left(63.9 \%, t_{36}=6.273, P=3 \times 10^{-7}\right)$ and without resort to guessing strategies, although performance was lower overall when children were tested in the more distracting classroom environment $\left(F_{1,55}=5.56, P=.022\right.$, Figure $\left.2 b\right)$. Children's performance of approximate symbolic arithmetic in this experiment was 
correlated with their performance on a test of mastery of the school's curriculum, although the specificity and the causal nature of this relationship remain to be established. Capacities for symbolic arithmetic evidently are available to children across a range of social and economic backgrounds, and they can be elicited in classroom as well as laboratory settings.

What processes underlie children's symbolic approximate arithmetic? We first considered whether children might have learned some symbolic addition facts and used their fact knowledge to solve the present problems. Contrary to this possibility, children failed to provide the exact solution to the present problems, to solve those problems when the comparison quantity was altered so as to require an exact representation of number, or to solve the problems through rounding strategies allowing for single-digit addition or comparison. Children's approximate arithmetic performance evidently does not depend on knowledge of exact number.

We therefore considered whether children draw on nonsymbolic, approximate number representations in solving these symbolic problems. Nonsymbolic number representations show three signature properties in human adults, children, infants, and non-human animals. First, comparison, addition and subtraction are subject to a ratio limit: accuracy falls as the ratio of the numbers to be compared approaches $1^{48916}$. Second, addition is as accurate as comparison: when adults or children sum two arrays and compare the result to a third array, they perform as accurately as when they compare the third array to a single array with the numerosity of the sum ${ }^{9}$. Third, subtraction is less accurate than comparison ${ }^{9}$. If children use nonsymbolic number representations to perform symbolic arithmetic, their performance should show the same signatures. 
To test for the ratio signature, we compared children's performance at each of the three ratios. Children's accuracy decreased as the ratio of the sum of the addends to the comparison set approached 1 (for laboratory-tested children, $F_{1,19}=14.92, P=2 \times 10^{-5}$, Figure $2 \mathrm{a}$; for school-tested children, $F_{1,36}=5.32, P=.027$, Figure $2 \mathrm{~b}$ ). The effect of ratio was the same for these symbolic problems as for problems presented nonsymbolically, ${ }^{8}$ and it did not depend on the differential use or effectiveness of strategies that serve as an alternatives to addition.

To test for the other two signatures, we presented a new group of children from the same population as those in the previous laboratory experiments with symbolic subtraction and comparison tasks (Figure $1 \mathrm{~b}$ and 1c). Children solved subtraction problems $\left(67.7 \%\right.$ correct, $t_{19}=5.55, P=7 \times 10^{-6}$; Figure $\left.2 \mathrm{c}\right)$ without basing their answers on exact number knowledge or on a range of alternative strategies and with a ratio limit on subtraction performance (Figure 2b). Performance on the comparison problems $\left(80.4 \%\right.$ correct, $\left.t_{19}=9.82, P=1 \times 10^{-10}\right)$ did not differ from performance on the symbolic addition problems $\left(F_{1,47}=2.20, P=.145\right)$ but was higher than performance on the symbolic subtraction problems $\left(F_{1,27}=16.58, P=.0004\right)$. Nevertheless, a direct comparison of performance on the symbolic addition and subtraction problems revealed no significant advantage for addition $\left(F_{1,46}=1.36, P=.250\right)$.

We have shown that children with no formal arithmetic instruction perform symbolic addition and subtraction with limits that reveal three signatures of the nonsymbolic arithmetic system: a ratio effect on accuracy, addition performance that is as accurate as direct comparison, and subtraction performance that is less accurate than comparison. These common signatures suggest that children recruit their nonsymbolic number knowledge when they confront new problems of approximate symbolic arithmetic. Once children have learnt the verbal counting system and mapped number words onto nonsymbolic representations of number, they spontaneously use 
nonsymbolic system processes to manipulate quantities presented symbolically. Recruitment of the nonsymbolic system therefore does not depend on instruction in arithmetic.

Arithmetic instruction in most elementary school curricula focuses primarily or exclusively on exact operations on small numbers. Most children take years to master the set of exact, single-digit addition and multiplication facts ${ }^{17}$, and mastery of these facts is vulnerable to fatigue or interference even in adults ${ }^{18}$. For this reason, the teachers in our school-based study were sceptical about our experiments and surprised both by their students' success and by their enjoyment of the tasks. Our findings suggest that the difficulty of learning and performing arithmetic stems from the demands of constructing and operating on representations of exact number: representations beyond the limits of precision of nonsymbolic number representations ${ }^{19}$. When the requirement for exact precision is removed, children are able to solve symbolic addition and subtraction problems even in the absence of relevant instruction. A wide socioeconomic range of children, tested in varying settings, used nonsymbolic number representations to solve symbolic problems involving quantities that fell within the scope of those preexisting representations. Arithmetic instruction may be enriched by building on this competence.

\section{Methods summary}

In the first addition experiment, five-year-old children $(n=20)$ were given verbal problems with no visual accompaniment in which the comparison quantity differed from the sum by a 2:3 ratio. In the remaining addition experiments, children were presented with verbal problems accompanied by images of characters with sets labelled by Arabic numerals (Figure 1a). The comparison numbers were varied to present problems at 3 ratios: 4:7, 4:6, and 4:5. Children in the second experiment $(n=20)$ were 
drawn from a highly educated community and tested in the laboratory; those in the third experiment $(\mathrm{n}=37)$ were drawn from a lower middle-class community and tested in school. In the final experiment, children $(n=29)$ were given tests of symbolic subtraction and comparison, using verbal problems and images (Figures $1 \mathrm{~b}$ and 1c). All types of problems used the same comparison quantities as the problems in past studies of nonsymbolic addition ${ }^{8}$. The problems were designed either to prevent or to allow tests for guessing strategies.

For each experiment, one-sample two-tailed t-tests compared performance to chance $(50 \%)$ and one-way linear-trend analysis of variance tested for the effect of ratio on performance. Performance on experiment 1 was compared to performance on a nonsymbolic addition $\operatorname{task}^{8}$ by a one-way (Experiment) between groups analysis of variance with age as covariate. Performance across experiments was compared by 2 (Experiment) by 3 (Ratio) mixed-factor analyses of variance. Subtraction and comparison performance were compared by a 2 (Condition) by 3 (Ratio) repeated measures analysis of variance.

A list of problems used in the experiments, a description of the tests of school achievement and their relation to performance of symbolic approximate arithmetic, and a description of all the tests for guessing strategies is given in the Supplementary Information at www.nature.com/nature).

1. Baroody, A.J. \& Dowker, A. The Development of Arithmetic Concepts and Skills: Constructing Adaptive Expertise (Erlbaum, Mahwah, NJ, 2003).

2. Geary, D. From infancy to adulthood: the development of numerical abilities. Eur. Child Adoles. Psy. 9, 11-16 (2000). 
3. Gallistel, C.R. \& Gelman, R. Preverbal and verbal counting and computation. Cognition 44, 43-74 (1992).

4. Dehaene, S. The Number Sense (Oxford Univ. Press, Oxford, UK,1997).

5. Lemer, C., Dehaene, S., Spelke, E. \& Cohen, L. Approximate quantities and exact number words: dissociable systems. Neuropsychologia 41, 1942-1958 (2003).

6. Hauser, M.D. \& Spelke, E.S. Evolutionary and developmental foundations of human knowledge: A case study of mathematics. in The Cognitive Neurosciences (ed Gazzaniga, M.), $3^{\text {rd }}$ vol,853-864 (MIT Press, Cambridge, MA, 2004).

7. McCrink, K. \& Wynn, K. Large number addition and subtraction by 9-month-old infants. Psychol. Sci. 15, 776-781 (2004).

8. Barth, H., La Mont, K., Lipton, J. \& Spelke, E.S. Abstract number and arithmetic in preschool children. Proc. Natl. Acad. Sci. USA 102, 14116-14121 (2005).

9. Barth, H., et al. Non-symbolic arithmetic in adults and young children. Cognition 98, 199-222 (2006).

10. Pica, P., Lemer, C., Izard, V. \& Dehaene, S. Exact and approximate arithmetic in an Amazonian indigene group. Science 306, 499-503 (2004).

11. Zur, O. \& Gelman, R. (2004). Young children can add and subtract by predicting and checking. Early Child. Res. Q. 19, 121-137 (2004).

12. Griffin, S. \& Case, R. Evaluating the breadth and depth of training effects when central conceptual structures are taught. Monogr. Soc. Res. Child.61, 83-102 (1996).

13. Lipton. J.S. \& Spelke, E.S. Preschool children's mapping of number words to nonsymbolic numerosities. Child Dev. 76, 978-988 (2005).

14. Le Corre, M., Van de Walle, G., Brannon, E.M. \& Carey, S. Re-visiting the competence/performance debate in the acquisition of the counting principles. Cognitive Psychol. 52, 130-169 (2006). 
15. Temple, E. \& Posner, M.I. Brain mechanisms of quantity are similar in 5-year-old children and adults. Proc. Natl. Acad. Sci. USA 95, 7836-7841 (1998).

16. van Oeffelen, M.P. \& Vos, P.G. A probabilistic model for the discrimination of visual number. Percept. Psychophys. 32, 163-170 (1982).

17. Ashcraft, M.H. \& Fierman, B.A. Mental addition in $3^{\text {rd }}, 4^{\text {th }}$, and $6^{\text {th }}$ graders. $J$. Exp. Child Psychol. 33, 216-234 (1982).

18. LeFervre, J.-A., Smith-Chant, B.L., Hiscock, K., Daley, K.E. \& Morris, J. Young adults' strategic choice in simple arithmetic: Implications for the development of mathematical representations. in The Development of Arithmetic Concepts and Skills: Constructing Adaptive Expertise (eds. Baroody, A.J. \& Dowker, A.) 203-228 (Erlbaum, Mahwah, NJ, 2003).

19. Feigenson, L., Dehaene, S. \& Spelke, E.S. Core systems of number. Trends Cogn. Sci. 8, 307 (2004).

Supplementary Information is linked to the online version of the paper at www.nature.com/nature.

Acknowledgements We thank Curren Katz and Raphael Lizcano for help with data collection. This work was supported by a ROLE grant from the National Science Foundation (to E.S.).

Author Information Reprints and permissions information is available at npg.nature.com/reprintsandpermissions. The authors declare no competing financial interests.

Correspondence and requests for materials should be addressed to C.K.G. (camilla.gilmore@nottingham.ac.uk) or E.S.S. (spelke@wjh.harvard.edu). 
Figure 1. Example problems of symbolic, approximate arithmetic testing (a) addition, (b) subtraction, and (c) comparison.

Figure 2. Five-year-old children's performance on approximate addition, subtraction, and comparison problems. (a) Children's performance of addition problems in which the sum differed from the comparison number at three ratios. Solid bars indicate performance in the present experiment with numbers presented as words and symbols $(n=20)$; dotted bars indicate performance in a previous study with the same numerical values presented nonsymbolically as dot arrays. ${ }^{8}$ (b) Children's performance of the same symbolic addition problems, administered in a classroom setting $(n=37)$. (c) Children's performance of equivalent symbolic subtraction and comparison problems $(n=29)$. Error bars give s.e.m. 
Methods

Verbal addition experiment. Twenty children ( 9 male, mean age 5 years 10 months, range 5:5 to 6:6) were asked questions of the form "If you had twenty-four stickers and I gave you twenty-seven more, would you have more or less than thirty-five stickers?" Eight questions involved large numbers (in the range 5 to 98) selected so that the sum was larger on half the trials and differed from the comparison number by a ratio of 2:3. Two control questions (not analysed) involving small numbers and familiar sums $(2+2$ vs. $20 ; 10+10$ vs. 5$)$ were included to maintain motivation and assure children were on task. The children received no feedback but general encouragement throughout.

Computer-based symbolic addition. Twenty children (11 male, mean age 5:10, range 5:4 to $6: 1$ ) were given arithmetical problems presented both visually on a computer screen (see Figure 1a) and verbally by the experimenter. Initially two characters appeared and were named on screen. On one example trial, the experimenter stated "Sarah has fifteen candies" as a bag displaying the appropriate Arabic numeral appeared above the character on the left. Next, a second bag displaying an Arabic numeral appeared above the same character and the experimenter stated "and she gets nineteen more". Finally, a different-coloured bag displaying an Arabic numeral appeared above the other character and the experimenter stated "John has fifty-one candies" and asked "Who has more?". Twenty-four problems presented large numbers in the range 5 to 58 , selected such that the sum was greater than the comparison number on half the trials and differed from it by one of three ratios--4:7, 4:6, and 4:5--on 8 trials each. Three control problems (not analysed) involved small numbers and familiar sums. The session was split into three blocks and the characters and items (candies, toys, cookies) changed between blocks to maintain the children's interest in the task. Children were encouraged to guess, were given no feedback, and received stickers after 
each block of trials. Following the completion of the task 12 children were asked two probe exact questions. They were shown again the stimuli for two questions which they had solved correctly and were asked "If Sarah has fifteen candies, and she gets nineteen more, how many does she have altogether?". The sessions were videotaped to allow children's response times to be measured.

Computer-based addition in a school setting. Thirty-seven children (15 male, mean age 5:11, range 5:5 to 6:5) were recruited from a public kindergarten in an area of mid- to low- socioeconomic status; $37.6 \%$ of the students in the school lived in families whose income level met the state's qualifications for free or reduced-price lunch. Children were tested during school hours, in the kindergarten hallway. The procedure was otherwise the same as the computer-based addition in the laboratory.

Computer-based subtraction and comparison. Twenty-nine children ( 9 male, mean age 5:11, range 5:4 to $6: 4$ ) completed first a subtraction task followed by a comparison task. The tasks were the same as in the preceding addition experiments except as follows. For the subtraction task, the experimenter said "Sarah has fifty-five candies" as the bag bearing an Arabic numeral appeared above the left-most character. Then a second bag then moved into view from behind the first and moved to the side as the experimenter said "and she gives twenty-one of the candies away". Animations were used because pilot-testing revealed that children had difficulty understanding a subtraction transformation when the operands were presented statically. The children were given 4 small-number practice trials (not analysed) followed by 24 experimental trials, matched to the addition trials of the computer-based addition studies so that the comparison values were the same and the difference in the computer-based subtraction was equal to the sum in the computer-based addition. Because the quantities involved in a subtraction problem are necessarily larger than those of an equivalent addition problem with the same result, a subset of the addition and subtraction problems were 
also matched for the numbers involved in the operation. Half the addition and subtraction problems used smaller vs. larger numbers, and the small subtraction problems were matched with the large addition problems for the size of the initial quantity (e.g. addition $20+16$; subtraction $20-8$ ). Trials were split into blocks as in the previous studies. After the last trial, 20 children were asked two exact subtraction questions "If Sarah has A candies and she gives B of them away, how many does she have left?".

For the comparison task, a red bag appeared above the first character and the children were told "Sarah has fifty-one candies", then a blue jar appeared above the second character and the children were told "Paul has thirty cookies", finally a red bag appeared above the third character and the children were told "John has thirty-four candies" and were asked "who has more candies, Sarah or John?". An irrelevant numerical quantity was presented between the numbers to be compared in order to equalize the presentation of number words across tasks. The children were asked 4 small-number practice trials (not analysed) followed by 12 experimental trials in which the two values to be compared matched the sums/differences and comparison numbers for 4 of the 8 problems at each ratio in the previous experiments. These trials were split into two blocks. 
a

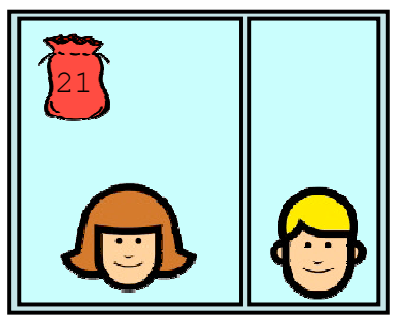

"Sarah has 21 candies."

b

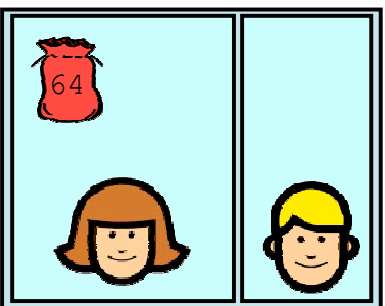

"Sarah has 64 candies."

C

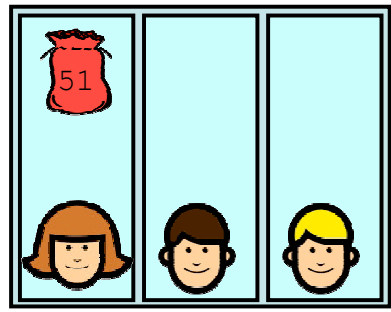

"Sarah has 51 candies."

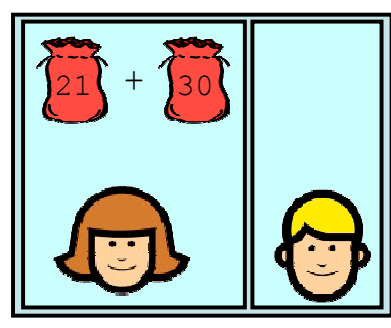

"She gets 30 more."

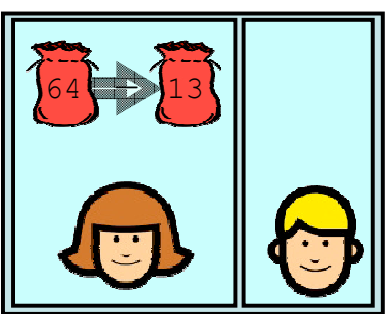

"She gives 13 of them away."

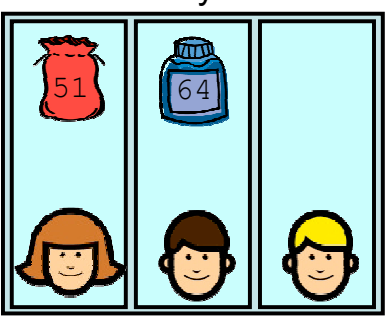

"Paul has 64 cookies."

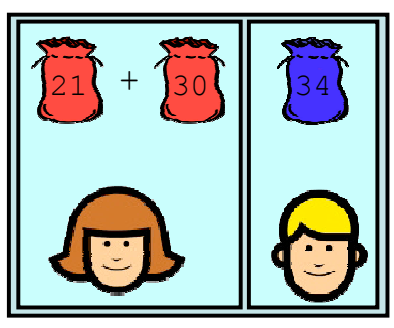

"John has 34 candies. Who has more?"

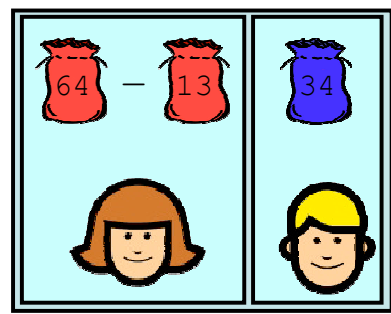

"John has 34 candies. Who has more?"

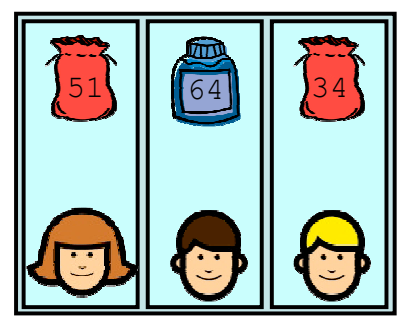

"John has 34 candies. Who has more candies, Sarah or John?" 
a

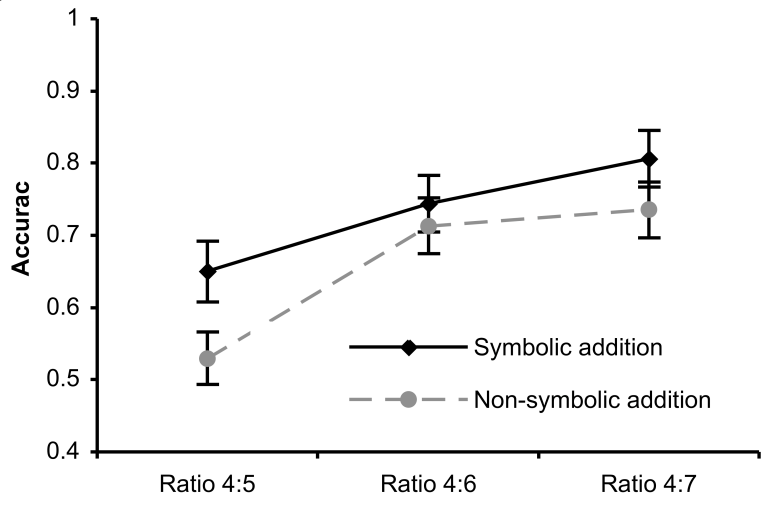

b
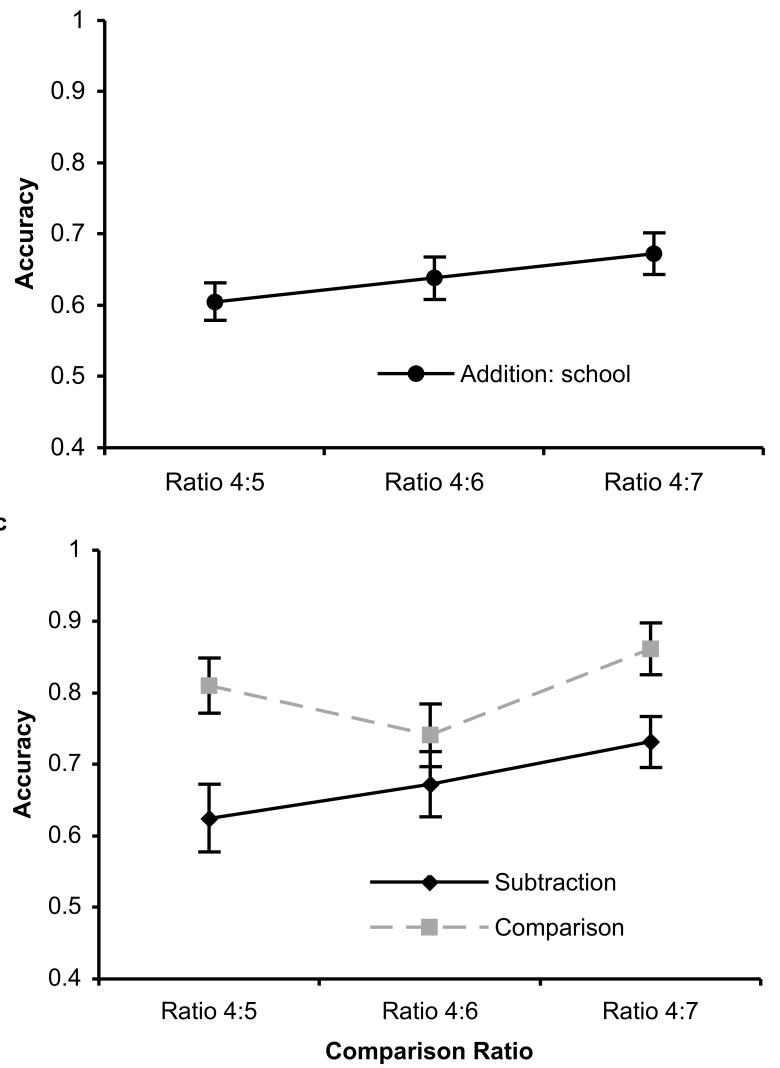